\title{
Database system design of casting process based on the Delphi technique
}

\author{
Zhao Hongli, Zhang zhishan
}

Zaozhuang vocational college of science and technology, Shandong Tengzhou 277500

Keywords: casting process; database system; CAD

\begin{abstract}
Through the Delphi technique combined with the application examples, this article processed design for the basic functions of the database system of casting process, through the three dimensional diagram of a casting, it processed draft Angle operation, minimum cast hole operation, the machining allowance operation, shrinkage operation four aspects to get the corresponding parameters, and has carried on the 3D drawing of module with the help of AutoCAD, realizing the combination with AutoCAD to the casting process parameters.
\end{abstract}

\section{Introduction}

With the development of computer technology, the application of computer technology in foundry industry is becoming more and more widely, such as casting process CAD, casting process $\mathrm{CAE}$ and so on, and casting process database is actually a kind of engineering databases, which is a member of the engineering database family, closely linked to other engineering databases. The commonality of casting process database remains to be strengthened, mainly because each manufacturer's situation is different, and there is also a very big difference between productions need to product, thus increases the difficulty for casting process database development. Judging an enterprise whether it has a strong competitive and long-term development space in the industry, just sees if the enterprise has set up a casting process database with comprehensive content, reasonable classification, scientific operation, perfect means and advanced technology.

\section{Database structure}

Casting process database system is divided into four modules, and their relationship structure is shown in figure 1.

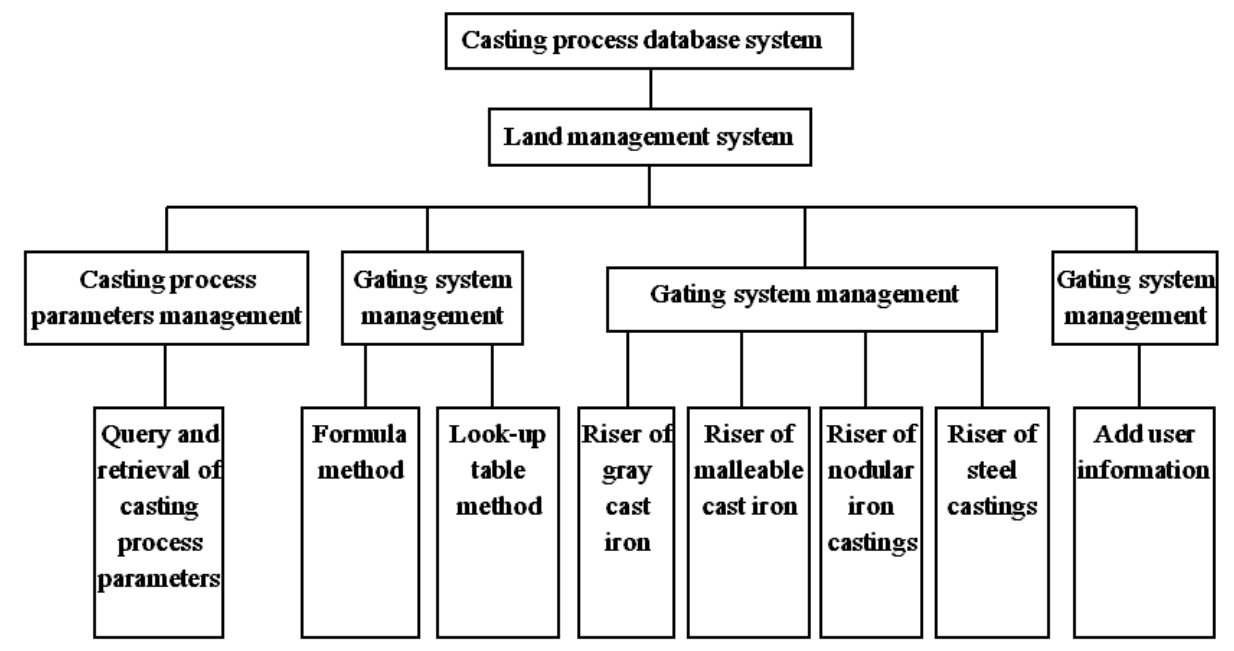

Figure 1 Relationship diagram of casting process database

After inputting the correct login information, the user can enter the system main interface, otherwise it will bring up a prompt dialog, pointing out your mistake information.

Inputting the correct information into the system, what displayed on the screen is the main interface of the system. The top of interface displays the casting process parameters management menu, the gating system management menu, feeding system management menu, system settings 
management menu and exit button. By clicking on these buttons and menus, the user can achieve its corresponding functions.

Click on the casting process parameters management in the upper left corner, under which it will conveniently display parameters drop-down menus such as: casting minimum wall thickness, casting minimum cast hole, the casting size tolerance and other parameters, and we can according to actual needs to make the next step.

After clicking on the query and retrieval button of casting process parameters, we should select casting minimum wall thickness in the drop-down menu, and then click, the casting minimum wall thickness interface will be displayed on the screen. Input the contents need to query in the query conditions, such as: " $\leq 200 \times 200$ ", then click "search" button, and then in the query results it will show the minimum wall thickness of different materials conform to the conditions.

Click on the casting shrinkage button, and enter into the casting shrinkage rate interface. Only need to input the query conditions in this interface, we can realize the query and retrieval, and even adding and deleting for material shrinkage rate. The bottom of the interface is the data controller, using the data controller we can browse data, also can add, modify, and delete data.

Button click gating system management button, there is a drop-down menu, which shows formula method and the look-up table method, we can select the corresponding query methods according to the actual needs.

\section{Demand analysis of database}

Sharing and managing information resources stably, conveniently and quickly are service purposes of the database. Standard data and empirical data are stored in the casting process database, with a huge number, but in the dynamic process of technological design, it will produce large amounts of data, the data also need to save in time and modify again and again. Therefore, able to quickly query complex data type definition, able to preserve and to deal with dynamic data in a timely manner, are the basic requirements to the database management system (DBMS). Accurately, timely and effectively meeting the needs of the user to the database, is the basic goal of database design. As shown in figure 2, the user can not only can browse and query the information in the data source in time, also can timely get the optimized scheme of defect analysis.

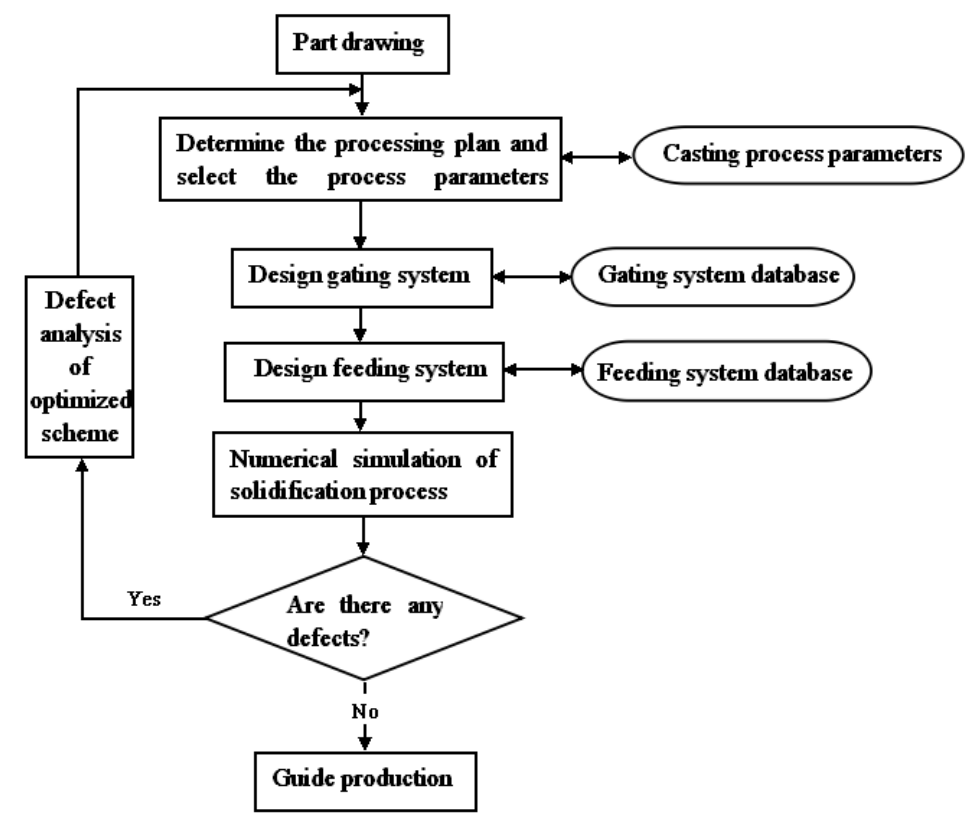

Figure 2 Flow diagram of optimized scheme of defect analysis 


\section{Instance operations}

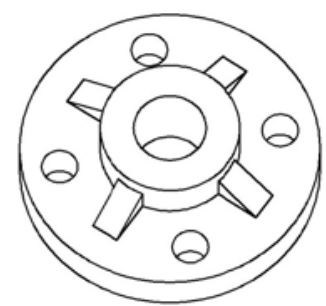

Figure 3 Three-dimensional diagram (before operation)

The figure 3 is the three dimensional diagram of a casting, according to the established database now carrying out the following operations for parts drawings:

[1] Draft Angle operation: operation object - cylindrical parts and oblique rib (increasing sizes in the bottom).

[2] Smallest cast hole: operation object - four round holes.

[3] Machining allowance operation: operation object - parts base.

[4] Shrinkage operation: operation object - the entire casting.

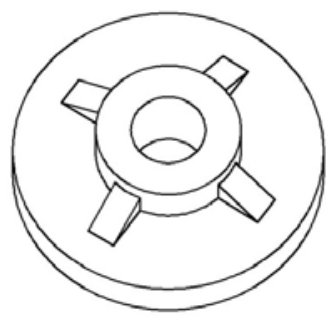

Figure 4 Three-dimensional diagram (Has been operating) observation)

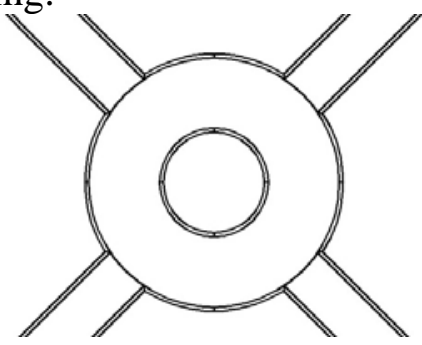

Figure 5 Part of enlarged figure (draft Angle

According to the comparison of figure 3 and figure 4 shows that after the operation, the hole in figure 3 has disappeared, it is because the hole size is less than the minimum cast hole, and should not be cast out when casting, but after mechanical processing to complete. In order to facilitate observation, figure 5 is the enlarged figure of circular truncated cone and oblique rib, so as to observe their draft Angle sizes, to prove to the realization of the draft Angle.

\section{Conclusion}

This chapter has introduced the basic function of casting process database system management, such as casting process parameters management module, gating system management module, etc., and has carried on the simple introductions for the corresponding modules. Through draft Angle operation, minimum cast hole operation, machining allowance operation, shrinkage operation four aspects to the three dimensional diagram of a casting to get the corresponding parameters, this chapter conducted 3D drawing to module using AutoCAD, realizing the combination the casting process parameters and AutoCAD, which embodies the visualization of casting process design.

\section{Reference}

[1] Tang Xinzi. Research and development of CAPP database system based on the C_S structure [D]. Xiangtan: Xiangtan University, 2006

[2] Wei Ping. Research and development of tool management system based on C/S and B/S mixed structure [D]. Xi 'an: Northwestern Polytechnical University, 2004

[3] Li Jun. Use ADO in Delphi6 application development [J]. Journal of Shaanxi Institute of Technology, 2003(1): 35-37 